\title{
Anomaly detection in spatiotemporal data via regularized non-negative tensor analysis
}

\author{
Chaoguang Lin ${ }^{1}$. Qiuhan Zhu' ${ }^{1}$ Shunan Guo' ${ }^{1}$. \\ Zhuochen Jin' ${ }^{1}$ Yu-Ru Lin ${ }^{2}$. Nan Cao ${ }^{1}$
}

Received: 17 November 2016 / Accepted: 15 March 2018 / Published online: 29 March 2018

(C) The Author(s) 2018

\begin{abstract}
Anomaly detection in multidimensional data is a challenging task. Detecting anomalous mobility patterns in a city needs to take spatial, temporal, and traffic information into consideration. Although existing techniques are able to extract spatiotemporal features for anomaly analysis, few systematic analysis about how different factors contribute to or affect the anomalous patterns has been proposed. In this paper, we propose a novel technique to localize spatiotemporal anomalous events based on tensor decomposition. The proposed method employs a spatial-feature-temporal tensor model and analyzes latent mobility patterns through unsupervised learning. We first train the model based on historical data and then use the model to capture the anomalies, i.e., the mobility patterns that are significantly different from the normal patterns. The proposed technique is evaluated based on the yellow-cab dataset collected from New York City. The results show several interesting latent mobility patterns and traffic anomalies that can be deemed as anomalous events in the city, suggesting the effectiveness of the proposed anomaly detection method.
\end{abstract}

Keywords Tensor analysis · Anomaly detection - Outlier detection · Urban computing · Traffic analysis

\footnotetext{
Responsible editor: Jieping Ye.

$凶 \quad$ Nan Cao

nan.cao@tongji.edu.cn

Yu-Ru Lin

yurulin@pitt.edu

1 Intelligent Big Data Visualization Lab, Tongji University, Shanghai, China

2 University of Pittsburgh, Pittsburgh, USA
} 


\section{Introduction}

The high availability of transportation data such as taxi archives provides great opportunities for understanding people's mobility patterns in a city. Despite the analysis performed for understanding the majority moving trend such as (Yuan et al. 2012), detecting and understanding regions with anomalous mobility patterns attract more and more attention especially in the big cities such as New York where traffic congestion is always a big transportation issue. Understanding those anomalous traffic patterns can help with a better management of the city.

The above problem lies in the domain of anomaly detection which has been extensively studied in the field of data mining and machine learning. Many techniques have been proposed (Chandola et al. 2009) and applied to various application domains. However most of the existing techniques fail to provide a clear decomposition of the data to reveal the potential factors that best capture, describe, or affect the anomalous behaviors. These factors are usually critical for the interpretation of the analysis results and can be helpful for analyst to control and prevent the undesired anomalies. For example, in the above problem, to detect anomalous mobility patterns based on the transportation data, we need to examine the data from multiple perspectives such as space, time, traffic volumes, and their relationships. Matrix-based methods such as PCA, though proved to be powerful in many areas, cannot deal with multi-way data. Some empirical evidence (Fanaee-T and Gama 2016a) has shown the superiority of tensor-based methods over traditional matrix-based methods. On this occasion, tensor-based methods are appropriate since the tensor could store the natural multi-dimensional relationship of the data. Tensor-based anomaly detection methods (Fanaee-T and Gama 2016a) are able to decompose a multi-way tensor into information factors and reveal their relationships based on probability models. However, none of the existing tensor-based techniques are specifically developed to detect anomalous traffic patterns, such as sudden increase in the volume of incoming or outgoing people of a particular region or irregular traffic flow among several regions.

In this paper, we propose a novel anomaly detection technique based on tensor decomposition for spatiotemporal data. The proposed technique follows unsupervised learning procedure in which a model is trained based on samples showing normal traffic patterns. Later, the extracted patterns are used for detecting anomalous transportation regions that significantly differ from the normal cases. In particular, we first segment a city into several regions and extract traffic features from each region for analysis. A three-way tensor, spatial-feature-temporal, is prepared based on the features and decomposed into three key information facets that describe how the latent mobility patterns are distributed in dimensions of space, feature and time, respectively. Then local outlier factor is calculated based on the decomposition results and the findings are interpreted in both spatial and temporal context. This paper has the following key contributions:

- We propose a novel framework, $T B A D$, based on tensor decomposition to localize anomalies in dynamic traffic system. Our method can localize anomalous regions in a given time interval. 
- We give an intuitive interpretation of the latent patterns extracted from decomposition.

- We evaluate the performance of our framework on real-world datasets. All our experiments show the effectiveness and consistency of our framework in localizing spatiotemporal anomalous events.

The rest of this paper is organized as follows. We first discuss related work in Sect. 2, followed by preliminary knowledge and notations in Sect. 3. Section 4 introduces the model and data processing pipeline for anomaly detection. In Sect. 5, we describe an empirical evaluation of the proposed method through real-world data. Finally, we conclude with a summary and future directions in Sect. 6.

\section{Related work}

In this section, we review techniques that are most related to our work, including classic anomaly detection algorithms, tensor-based anomaly detection and spatiotemporal event detection.

\subsection{Classic anomaly detection algorithms}

Anomaly detection has been extensively studied during the past decades, many classic techniques have been proposed (Chandola et al. 2009; Jiang and Cui 2016). In general, these techniques can be categorized into several types such as statistic methods, classification-based methods, spectral-based methods and so on. Although each type of these methods has its own advantages and disadvantages, all these techniques can only produce detecting results without reasonable interpretation of the anomaly, which makes it difficult for people to understand and validate the result. Besides, most of the methods are matrix-based and are not able to tackle the multi-dimensional data. Spectral-based method is considered as one of the most appropriate methods for tackling high dimensional data, which automatically performs dimensionality reduction and can also be used as a preprocessing step followed by other methods in the projected space. We thus consider using a tensor-based method to perform dimensionality reduction and also impose non-negativity constraint to make the result interpretable. In particular, we use the non-negative $\mathrm{CP}$ decomposition as a spectral method for dimensionality reduction and interpretation.

\subsection{Tensor-based anomaly detection}

Recently, Hadi et al. did a comprehensive review of the tensor-based anomaly detection techniques, most of which were developed beyond the scope of computer science (Fanaee-T and Gama 2016a). Here we focus on those tensor based methods that are most related to our work. 


\subsubsection{Supervised model}

Supervised tensor-based anomaly detection techniques have been developed based on dimensionality reduction (Prada et al. 2012a, b; Tork et al. 2012; Wang et al. 2014; Fanaee-T and Gama 2016b; Bai et al. 2013), classification (Tao et al. 2005; Kotsia et al. 2012; Rendle 2012), and prediction (Zheng et al. 2014; Zhao et al. 2015; Matsubara et al. 2012; Bahadori et al. 2014; Thai-Nghe et al. 2010). Our work is inspired by dimensionality reduction and feature extraction based approaches.

\subsubsection{Semi-supervised model}

Most of the semi-supervised models are designed for real-time anomaly detection and can be divided into two categories (Fanaee-T and Gama 2016a). Methods in both categories use normal data (i.e., positive samples) to construct a tensor and use the decomposition results as a baseline. Those decomposition results based on the testing data however failed to align with the baseline are considered as anomalies. Methods in the first category statistically test the null hypothesis such as Nomikos and MacGregor (1994) and Tian et al. (2009). Methods in the second category compare the differences between the baseline and the testing data based on the eigenvectors and eigenvalues of the factor matrices (Fanaee-T and Gama 2014, 2015). For example, Fanaee-T and Gama (2015) proposed a novel approach called EigenEvent. They generated a dynamic baseline tensor (Space Features Time) from historical data and process the coming time window into a two-dimensional matrix (Space Features). Then the matrix and the baseline tensor were decomposed into subspace and then matched with the eigenvectors and eigenvalues. Anomalous time windows were detected when the angle between the matrix's eigenvector was higher than expected or the ratio of matrix's eigenvalue to the baseline eigenvalue was higher than expected.

However, it is sometimes impossible for us to get labelled data especially in realworld problem. Thus our work mainly focus on unsupervised tensor-based model.

\subsubsection{Unsupervised model}

Most unsupervised tensor-based models used in anomaly detection (Papalexakis et al. 2012, 2014; Mao et al. 2014; Gauvin et al. 2014) plot the factor matrices obtained from the tensor decomposition and the anomalies are manually identified by human experts based on the plotting results. For instance, Papalexakis et al. (2014) used a three-way ("user-venue-time") tensor and detected anomalous components by plotting each component's values on each mode and found the most anomalous components by analyzing the images. In addition, Mao et al. (2014) applied CP decomposition on a three-way ("sourceIP-targetIP-time") tensor model to detect malicious network behavior. Gauvin et al. (2014) proposed a plot-based detection of the community-activity structure of temporal networks. Papalexakis et al. (2012) proposed a novel parallelizable tensor decomposition method called PARCUBE. They also showed some plot-based detection results based on PARCUBE. All these techniques follow a similar procedure in which the tensor is decomposed and the results are plotted for human experts to explore. Different from these methods, we propose an unsupervised model, 
which implements automatic anomaly detection after decomposition to discover the most suspicious spatial regions to users for a detailed inspection, which is more precise and efficient.

In addition, there are some related work (Sun et al. 2006, 2008; Shi et al. 2015) for online anomaly detection using tensor subspace analysis. These methods use reconstruction error as the metric for detecting anomaly. Although STA (Streaming Tensor Analysis) (Sun et al. 2006) is an efficient tool for tackling tensor stream, reconstruction model lacks some intuitive interpretation of the anomaly and may suffer a lot from the instability of real-world data. Our method can be used in online detection (see Sect. 4.2) and the non-negativity constraint makes it possible to interpret and understand the latent patterns (see Sect. 5.1).

We compare our work with two most relevant techniques as follows. Prada et al. (2012a, b) proposed a technique, in which PARAFAC was used to decompose a threeway ("space-time-frequency") tensor based on the normal samples. Then the derived time factor matrix was trained via $k \mathrm{NN}$. And features used in $k \mathrm{NN}$ were the latent components derived from the tensor decomposition. However, the aim of their work was detecting anomaly in engineering structures which is different from our application. Thus, their techniques cannot directly apply to our problem. Differently, our method is designed to capture the temporal dynamics of the transportation data. Some interpretable latent patterns are extracted, which better illustrates the decomposition results and helps understand the anomaly. Tork et al. (2012) applied a Tucker3 decomposition for discovering abnormal users in an IP/TV network based on the latent components that are derived from the tensor decomposition, but the analysis results are difficult to interpret. Our work use non-negative PARAFAC decomposition to derive latent mobility pattern based on real-world regions on map, which is more intuitive. In addition, a simple map visualization (See Sect. 5.1) is used for analysis interpretation, inspired by some visual techniques designed for time-series data (Xie et al. 2014; Xu et al. 2017) and taxi trajectories (Liu et al. 2017; Weng et al. 2018).

\subsection{Spatiotemporal anomaly detection}

There are techniques designed specifically for anomaly detection in spatiotemporal data. For example, monitoring the gas sensor networks (Wang et al. 2008) and detecting anomalies in the spatiotemporal network data (Young et al. 2014; Zhang et al. 2017; Paschalidis and Smaragdakis 2009). However, these techniques are designed for a targeted domain based on many assumptions in that specific field. In comparison, Graph-TSS proposed by Liu et al. (2016) utilizes the latent semantics of textual information for spatial event detection. This work performs experiments using Twitter data and can effectively detect several anomalous events such as Argentina civil unrest events. However, it is a graph-based method which produces the most anomalous subgraph from the network. Thus it may not detect some small events that only cause a few number of nodes changed. This problem also comes with EventTree (Rozenshtein et al. 2014). Although it is a benchmark in spatiotemporal field, it focuses more on the trajectories of big events. Since some local events may not influence the neighbor areas, they are difficult to find when using EventTree because no anomalous sub-graph 
Table 1 Notations

\begin{tabular}{ll}
\hline Notation & Definition \\
\hline $\mathcal{X}, \mathrm{X}, \mathrm{x}, x$ & Tensor, matrix, column vector, scalar \\
$\mathrm{X}(:, \mathrm{i})$ & $i$-th column of $\mathrm{X}$ \\
$\mathrm{X}(\mathrm{i},:)$ & $i$-th row of $\mathrm{X}$ \\
$\|\cdot\|$ & Frobenius norm \\
$\circ$ & Outer product \\
$X_{(n)}$ & Mode-n matricization of tensor $\mathcal{X}$ \\
$\otimes$ & Kronecker product \\
$\odot$ & Khatri-Rao product \\
\hline
\end{tabular}

is formed. Yet, Our method compares each region's behavior to its normal case. Even the anomaly has little influence on nearby regions, it will be detected once it behaved much different from its normal behavior. In addition, many visual techniques have been proposed to facilitate analyzing spatiotemporal data (Liu et al. 2014; Sun et al. 2013, 2017a, 2017b; Xia et al. 2016; Wu et al. 2018).

\section{Background and preliminaries}

Here we list all necessary background knowledge for tensor decomposition and our algorithm framework $T B A D$. Table 1 provides an overview of the notations we use.

The Kronecker product of two matrices $\mathrm{A} \in R^{I \times J}$ and $\mathrm{B}$, denoted by $\otimes$ is defined as Kolda and Bader (2009):

$$
A \otimes B=\left(\begin{array}{cccc}
a_{11} B & a_{12} B & \cdots & a_{1 J} B \\
a_{21} B & a_{22} B & \cdots & a_{2 J} B \\
\vdots & \vdots & \ddots & \vdots \\
a_{I 1} B & a_{I 2} B & \cdots & a_{I J} B
\end{array}\right)
$$

For two matrices $A=\left(a_{1}, a_{2}, \ldots, a_{k}\right), B=\left(b_{1}, b_{2}, \ldots, b_{k}\right)$ with the same number $k$ of columns, their Khatri-Rao product, denoted by $\odot$, is defined as Kolda and Bader (2009):

$$
A \odot B=\left(a_{1} \otimes b_{1}, a_{2} \otimes b_{2}, \ldots, a_{k} \otimes b_{k}\right)
$$

\subsection{Tensor}

A tensor, denoted by $\mathcal{X}$, is a multi-dimensional array, which is an extensional concept of matrix. In general, a N-way tensor(or Nth-order tensor) is a tensor of $\mathrm{N}$ dimensions. In particular, a zero-way tensor is a scalar, a one-way tensor is a vector and twoway tensor is a matrix. A $\mathrm{N}$-way tensor $\mathcal{X} \in \mathcal{R}_{+}{ }^{I_{1} \times I_{2} \times \cdots \times I_{N}}$ has $\mathrm{N}$ ways with the dimensionality of $I_{1}, I_{2}, \ldots, I_{N} . R_{+}$means all the elements of $\mathcal{X}$ are non-negative, which are commonly used in real-world applications. 


\subsection{Tensor decomposition}

Given a tensor $\mathcal{X}$, the $\mathrm{CP}$ decomposition (or PARAFAC decomposition) factorizes the tensor into a sum of rank-one tensors (Kolda and Bader 2009). For example, given a three-way tensor $\mathcal{X} \in \mathcal{R}^{I \times J \times K}$, it can be written as Kolda and Bader (2009):

$$
\mathcal{X} \approx \sum_{r=1}^{R} a_{r} \circ b_{r} \circ c_{r}=\llbracket A, B, C \rrbracket
$$

where $\mathrm{R}$ is a positive number and $a_{r} \in \mathcal{R}^{I}, b_{r} \in \mathcal{R}^{J}, c_{r} \in \mathcal{R}^{K}$ for $\mathrm{r}=1,2, \ldots, \mathrm{R}$. PARAFAC decomposition is usually represented in its matrix form $\llbracket A, B, C \rrbracket$ (Kolda and Bader 2009), where the columns of matrix $A, B, C$ are the $a_{r}, b_{r}, c_{r}$ vectors. In particular, $A=\left(a_{1}, a_{2}, \ldots, a_{R}\right), B=\left(b_{1}, b_{2}, \ldots, b_{R}\right), C=\left(c_{1}, c_{2}, \ldots, c_{R}\right)$.

As the symbol 'o' represents outer product of vectors, each element of the tensor $\mathcal{X}$ can be also written as:

$$
\mathcal{X}_{i j k} \approx \sum_{r=1}^{R} A_{i r} B_{j r} C_{k r}
$$

In this work, we use the non-negative $\mathrm{CP}$ decomposition in our framework, since it admits a very intuitive interpretation of its latent factors, and non-negativity is inherent to the data being considered.

\subsection{Local outlier factor}

The local outlier factor (LOF) is a classic anomaly detecting algorithm (Breunig et al. 2000). It detects anomalous data points by comparing each point's density with its k-nearest neighbors. LOF algorithm assigns scores of being an outlier to each data point. The score is called the local outlier factor(LOF) of a data point. Data points with high LOF value have less local densities than their k-nearest neighbors and are of great probability to represent outliers. A LOF-value around 1 implies that the point's density is similar to its neighbors, which is of great probability to declare that it is not an outlier. A LOF-value below 1 implies the point has lower density than its neighbors, which also indicates it is not an outlier.

However, it is not time-evolving and may face computational difficulties when dimensionality increases. Thus, we preprocess the data points before using LOF algorithm, which is illustrated in Sect. 4 and the result shows a significant improvement over pure LOF algorithm, which is discussed in Sect. 5.

\section{TBAD: a tensor-based spatiotemporal anomaly detection method}

We illustrate the pipeline of our algorithm model in Fig. 1a. The procedure consists of four steps. First, we formulate a set of region-feature matrices in consecutive time slices and build up a region-feature-time tensor. Second, we apply non-negative CP 
(a)

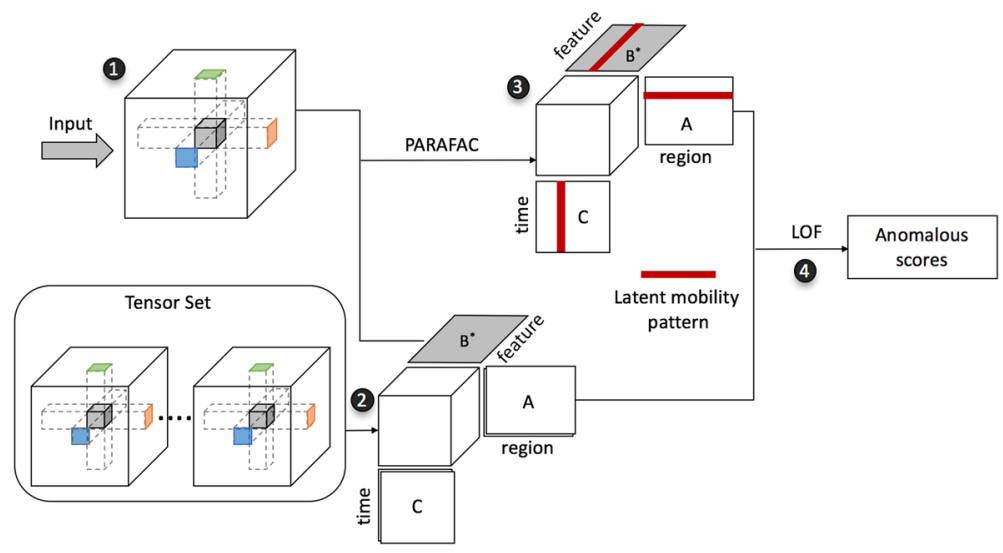

(b)
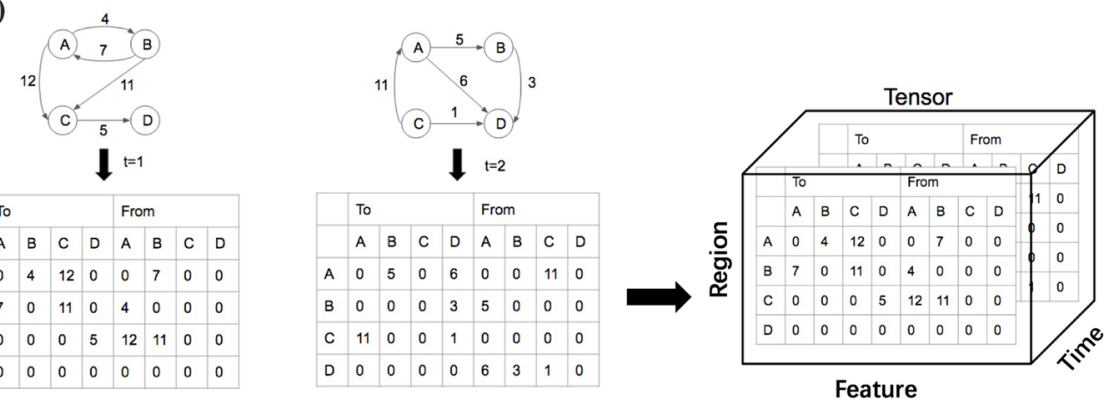

Fig. 1 System description. a Pipeline, $\mathbf{b}$ tensor formulation

decomposition on the tensor and extract latent mobility patterns from factor matrix $B^{*}$. Third, we decompose each upcoming tensor with respect to the former latent patterns $\left(B^{*}\right)$ and capture their dynamic distribution on temporal and spatial dimension. Finally, we compare each region's co-occurrence of latent patterns with its historical distribution. Anomalous regions often hold a different distribution of latent patterns and we can use the LOF algorithm to find them. Here we assume that anomalous regions are those with different traffic patterns compared to their normal historical situation. In addition, we can also use other classic anomaly detection algorithms such as one-class SVM (Chen et al. 2001) rather than LOF to detect anomaly.

\subsection{Model formulation}

As we discussed above, a tensor can store multi-dimensional information. Thus, we use a tensor to represent traffic flow in a period of time. Suppose that there are $K$ features to measure a region and a time interval divided into $M$ smaller time slices, we can construct a tensor $\mathcal{X} \in \mathcal{R}^{N \times K \times M}$ to indicate a spatiotemporal information, which represents the feature variances of different regions over time. In particular, the element $\mathcal{X}_{i j k}$ denotes the value of $\mathrm{j}$-th feature in $\mathrm{i}$-th region during the period of $\mathrm{k}$-th time slice. Note that there are two notions in time dimension. First, the tensor is built 
over a period of time(e.g., a day, a week or a year). The time interval is further divided into several fixed time slices, of which the granularity is determined according to the length of the time interval. For example, the length of the time slices can be set to one hour when the interval is a day, or a day when the interval is a week. In our experiment, the interval is a day and the length of time slices is $2-\mathrm{h}$.

The features used in the tensor are selected according to different data sources. The following describes the situation of tensor formulation using yellow taxi data in NYC. Suppose that the number of region is $N$, which is pre-determined. Then we use $2 N$ features to measure the incoming and outgoing flow of a certain region. The first $N$ features indicate the number of taxi trips leaving region- $i$. And the next $N$ features indicate the number of trips entering region- $i$. To be specific, for region- $i$, $\mathcal{X}[i, j, k]$ denotes the number of trips from region- $i$ to region- $j$ in $k$-th time slice and $\mathcal{X}[i, N+j, k]$ denotes the number of trips from region- $j$ to region- $i$ in $k$-th time slice, respectively. In particular, both $\mathcal{X}[i, i, k], \mathcal{X}[i, N+i, k]$ denotes the number of trips traveling inside region- $i$ (one of which is omitted in analysis to avoid redundancy). Figure $1 \mathrm{~b}$ shows an numerical example of our proposed tensor formulation method.

\subsection{Extracting basic mobility patterns}

With the large volume of historical traffic data, our goal is to find several basic mobility patterns which can be used to represent normal traffic behavior. In other words, a region's traffic pattern can be interpreted as linear combination of the former basic patterns. In this case, we can interpret a region's traffic patterns with the interpretation of the basic patterns. Technically, we are going to produce a factor matrix $B^{*}$ to extract basic mobility patterns in a city. We first get all the tensors $\mathcal{X}_{i}(\mathrm{i}=1, \ldots, \mathrm{n})$ to be trained and $B^{*}$ is calculated by:

$$
B^{*}=\underset{A_{i}, C_{i}, B^{*} \geq 0}{\operatorname{argmin}} \sum_{i=1}^{n}\left(\left\|\mathcal{X}_{i}-\llbracket A_{i}, B^{*}, C_{i} \rrbracket\right\|^{2}+\alpha\left\|A_{i}-A_{i-1}\right\|^{2}+\beta\left\|C_{i}-C_{i-1}\right\|^{2}\right)
$$

$B^{*}$ is the feature-component (basic mobility pattern) factor matrix. We suppose $B^{*}$ to be the common factor matrix of all the training tensors. Therefore, it stores the most common relationship between feature and basic traffic patterns and $A_{i}, C_{i}$ matrices capture the dynamic spatial and temporal distribution of basic patterns. Thus, $B^{*}$ can represent the most general basic mobility patterns on the training set.

The coefficients $\alpha, \beta$ here are used to control smoothness on factor matrices $A_{i}$ and $C_{i}$, which aims to eliminate the anomaly in the training set. Since the training set is not labelled, we suppose that imposing smoothness can eliminate the influence of the anomalies on the extracted latent patterns. The values of the two coefficients are set according to the specific situations.

It is easy to illustrate how this can be used in online situation. When the latest tensor $\mathcal{X}_{n+1}$ arrives, we can solve the optimization problem (2) by applying $\mathcal{X}_{n+1}$ to gain a new $B^{*}$. As time evolves, we can replace the oldest tensor $\mathcal{X}_{1}$ with the latest tensor $\mathcal{X}_{n+1}$ when solving (2) to save computation time and storage space. 
Besides, when dealing with time dependent models, different time intervals are not treated equally. In general, latest ones are more important than the ones in the past. To achieve this goal, additional coefficients can be added to the objective function.

$$
B^{*}=\underset{A_{i}, C_{i}, B^{*} \geq 0}{\operatorname{argmin}} \sum_{i=1}^{n} \gamma_{i}\left\|\mathcal{X}_{i}-\llbracket A_{i}, B^{*}, C_{i} \rrbracket\right\|^{2}
$$

$\gamma_{i}$ controls the importance of $i$ th-time interval. For simplicity, the experiments in our work still use (2) as objective function.

\subsection{Factorizing upcoming tensor}

With the factor matrix $B^{*}$ we got in Sect. 4.2, we apply non-negative CP decomposition to the upcoming tensor $\mathcal{X}$, which contains the latest traffic data to be detected, with the constraint that the $\mathrm{B}$ (feature-component) factor matrix is equal to $B^{*}$ :

$$
A, C=\underset{A, C \geq 0}{\operatorname{argmin}}\left\|\mathcal{X}-\llbracket A, B^{*}, C \rrbracket\right\|
$$

This is calculated under the assumption that the basic traffic patterns do not change dramatically in a short time. Thus we can use the same subspace to capture the relationship between feature and latent traffic patterns. With the same relationship between feature and latent patterns, we can then use the factor matrix $A$ and $C$ to capture the patterns' distribution on spatial and temporal dimension. In particular, matrix $A$ (region-component matrix) describes the patterns' co-occurrence on a certain region. For example, $A[i, k]$ captures the $k$-th pattern's occurrence on $i$-th region. The larger the value is, the more likely that region- $i$ behaves in tune with pattern- $k$. Matrix $C$ (time-component matrix) describes the pattern's temporal activity level. For example, $C[j, k]$ indicates the activity level of $k$-th pattern in $j$-th time slice. The larger the value is, the more traffic flow behaves in accord with pattern- $k$ in $j$-th time slice.

\subsection{Detecting anomaly by factor matrices}

According to Sects. 4.2 and 4.3, we got factor matrices $A_{i}(i=1, \ldots, n)$ for the training tensors and factor matrix $A$ for the detecting tensor. In particular, the row vector $A_{i}(k,:)$ in each factor matrix $A_{i}$ represents the patterns' historical co-occurrence on region- $k$. As discussed previously in the beginning of Sect. 4 , we hold the assumption that anomalous regions are those behave differently from their historical behavior. Thus we apply LOF algorithm to $A_{i}(k,:)(\mathrm{i}=1, \ldots, \mathrm{n})$ and $A(k,:)$ to detect whether the co-occurrence of the basic patterns on $k$-th region has changed and the lof-value is denoted as $l o f_{k}$. Moreover, $l o f_{k}$ will be a large value if the region shows such different traffic behavior that the co-occurrence of basic patterns changed dramatically. Thus we found regions' extent of anomaly by their lof-value. 


\subsection{Update rule}

We solve the above optimization problem (2) using block coordinate descent (Kim et al. 2014) and a multiplicative rule based on a tensor time series of the historical data. We treat $B, A_{t}, C_{t}(t=1, \ldots, n)$ as $2 n+1$ blocks and the update order is $A_{1} \rightarrow C_{1} \rightarrow A_{2} \rightarrow C_{2} \rightarrow \cdots A_{n} \rightarrow C_{n} \rightarrow B^{*}$. In particular, the update rules are:

1. solve $A(t), t=1,2, \ldots, n$

$$
\begin{aligned}
& A(t)=\underset{A \geq 0}{\operatorname{argmin}}\left(\left\|X_{t,(1)}^{T}-\left(C_{t} \odot B\right) A^{T}\right\|^{2}+\alpha\left\|A^{T}-A_{t-1}^{T}\right\|^{2}\right) \\
& =\underset{A \geq 0}{\operatorname{argmin}}\left\|X-F A^{T}\right\|^{2} \\
& \text { here } X=\left(\begin{array}{c}
X_{t,(1)}^{T} \\
\sqrt{\alpha} A_{t-1}^{T}
\end{array}\right), F=\left(\begin{array}{c}
C_{t} \odot B \\
\sqrt{\alpha} I
\end{array}\right) \\
& A_{j k} \leftarrow A_{j k} \frac{\left(X^{T} F\right)_{j k}}{\left(A F^{T} F\right)_{j k}}
\end{aligned}
$$

2. solve $C(t), t=1,2, \ldots, n$

$$
\begin{aligned}
& C(t)=\underset{C \geq 0}{\operatorname{argmin}}\left(\left\|X_{t,(3)}^{T}-\left(B \odot A_{t}\right) C^{T}\right\|^{2}+\beta\left\|C^{T}-C_{t-1}^{T}\right\|^{2}\right) \\
& =\underset{C \geq 0}{\operatorname{argmin}}\left\|X-F C^{T}\right\|^{2} \\
& \text { here } X=\left(\begin{array}{c}
X_{t,(3)}^{T} \\
\sqrt{\beta} C_{t-1}^{T}
\end{array}\right), F=\left(\begin{array}{c}
B \odot A_{t} \\
\sqrt{\beta} I
\end{array}\right) \\
& C_{j k} \leftarrow C_{j k} \frac{\left(X^{T} F\right)_{j k}}{\left(C F^{T} F\right)_{j k}}
\end{aligned}
$$

3. solve $B^{*}$

$$
\begin{aligned}
& B^{*}=\underset{B \geq 0}{\operatorname{argmin}} \sum_{t=1}^{n}\left\|X_{t,(2)}^{T}-\left(C_{t} \odot A_{t}\right) B^{T}\right\|^{2} \\
& =\underset{B \geq 0}{\operatorname{argmin}}\left\|X-F B^{T}\right\|^{2} \\
& \text { here } X=\left(\begin{array}{c}
X_{1,(2)}^{T} \\
X_{2,(2)}^{T} \\
\vdots \\
X_{n,(2)}^{T}
\end{array}\right), F=\left(\begin{array}{c}
C_{1} \odot A_{1} \\
C_{2} \odot A_{2} \\
\vdots \\
C_{n} \odot A_{n}
\end{array}\right) \\
& B_{j k} \leftarrow B_{j k} \frac{\left(X^{T} F\right)_{j k}}{\left(B F^{T} F\right)_{j k}}
\end{aligned}
$$

where $\mathrm{I}$ is the Identity matrix. The $X_{t,(n)}$ denotes the n-mode matricized version (Cichocki et al. 2009) of $X_{t}$. 


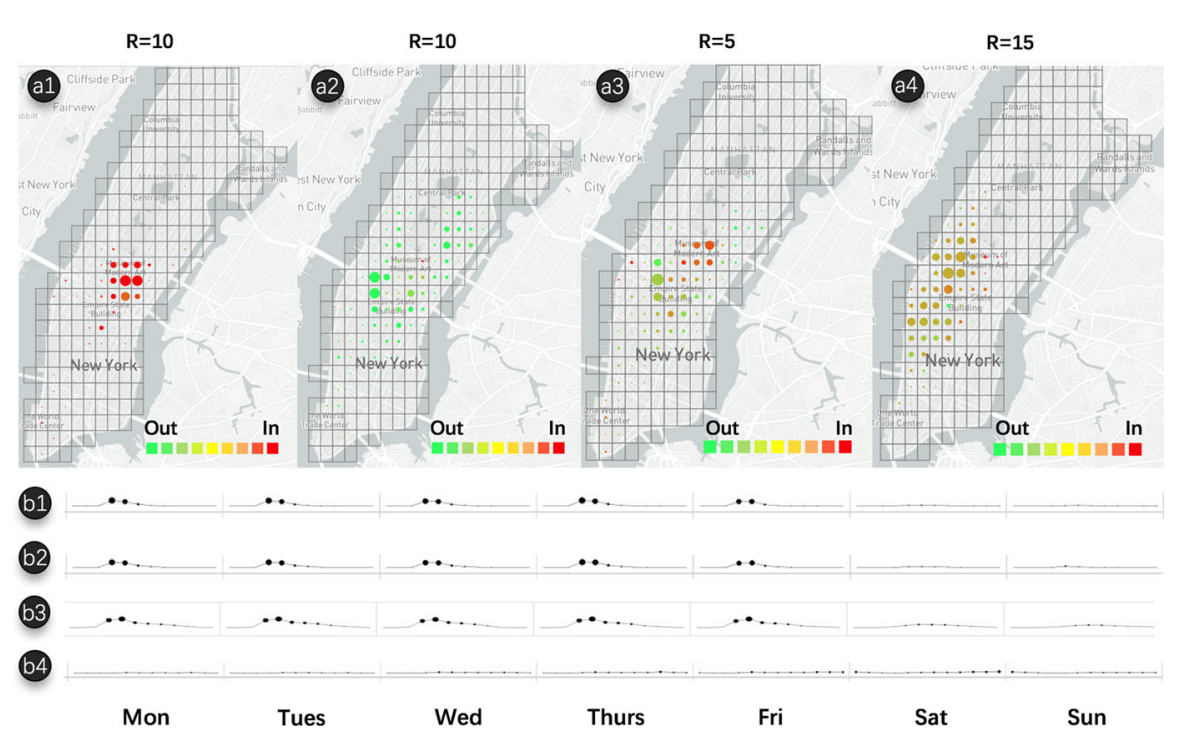

Fig. 2 Interpretation of latent patterns; (a1, a2, a3, a4) shows the spatial information of patters on the map and $(\mathrm{b} 1, \mathrm{~b} 2, \mathrm{~b} 3, \mathrm{~b} 4)$ reveals the pattern's temporal distribution respectively

\section{Empirical evaluation}

We applied $T B A D$ to real-world data sets in order to verify its accuracy. The experiment was conducted on New York City yellow taxi trip data in 2014. ${ }^{1}$ It contained around $3,000,000$ yellow cab trip data at New York City in 2014, which included trips' origin, destination, pick-up time, drop-off time and passenger count. We first used TBAD to find the anomaly region based on the traffic data. We used $R=5,10,15$ in our experiments and how to choose $\mathrm{R}$ are discussed in the next section. Special event corresponding to the anomaly region should be found to verify the correctness of $T B A D$. Then we compared the error rate of $T B A D$ with similar approach with untrained model and pure LOF.

\subsection{Interpretation of basic mobility patterns}

To illustrate how we interpret the extracted patterns, we visually summarize the spatial and temporal information by applying basic visual representations on the map of Manhattan. As shown in Fig. 2a1-a4, we place a grid on the top of the map to demonstrate the division of regions. The flow pattern of each region is encoded with a node inside the grid, with the size of node representing the volume of the flow, and the color representing the direction of the flow. A red-to-yellow-to-green color gradient is adopted here to indicate the average flow direction, with the red color indicating that the region contains more incoming trips and the green indicating more

\footnotetext{
1 https://data.cityofnewyork.us/view/gn7m-em8n.
} 
the outgoing trips. We further demonstrate the temporal distribution of the specific pattern (a1-a4) in Fig. 2b1-b4. As the length of time slices is 2-hour, we plot the temporal distribution of these patterns in each time slice. The size of nodes encodes the activity level so as to highlight time slices in which the pattern is more significant. Specifically, the size is set according to the $C_{i}(i=1, \ldots, n)$ factor matrices we got in Sect. 4.2. For example, the activity level of $k$ th-pattern in $j$ th-time slices in $i$ th-time interval is $C_{i}[j, k]$. Figure 2a1, a2 illustrate two distinct patterns when set $R=10$, and Fig. 2b1, b2 reveal their corresponding temporal characteristics. These patterns mainly appear around the center of Manhattan in 6 and 10 a.m. of the weekdays and disappear in weekends. This could reveal the morning peak of these regions, during which people flood into Manhattan Midtown (e.g., Rockefeller Center) to go to work.

To reveal the impact of parameter $R$ on the extracted patterns, we decrease and increase the value of $R$ to 5 and 15 as shown in Fig. 2a3, a4 respectively. When the value of $R$ is small, the extracted patterns are mostly aggregated, resulting in a mixture of incoming and outgoing flows, which makes it difficult to observe separately. On the contrary, if $R$ is too large, some extracted patterns will be less meaningful. For example, Fig. $2 \mathrm{~b} 4$ shows one of the temporal patterns when $R$ is set to 15 , which is inactive all the time, providing no useful information and should be considered as noise. Generally, patterns are more interpretative when the number of latent patterns increases. However, it will also produce more noise and less meaningful information. Thus, a properly chosen $R$ should provide us with more meaningful and less noisy patterns.

\subsection{Case study}

In order to locate the anomalous region easily, we divided the New York City into mesh-grid according to the longitude and the latitude. Then we computed anomaly score for each region. Regions with high anomaly scores were the anomalies we were trying to find. Following are several cases that $T B A D$ generate from the third-season data in 2014:

Electric Zoo Music Festival at Randall's Island Park Figure 3 shows the anomaly region detected by $T B A D$ on August 30 and 31. In Fig. 3, we have calculated the anomaly-score of the highlighted region on every Saturday. Most of the score was around 1, while the score on August 30th was 11.26. That value was significantly higher than the average score. So we considered August 30th as an anomalous date for that region. According to the speculation, we found that Electric Zoo music festival was held at Randall's Island park during that period.

Queen Mary 2(Ocean Liner) New York Arrival Figure 4 shows the extraordinary taxi flow between two regions shown on the map on September 27. The score(Day index 25 ) of those regions on September 27th was around 18.66, which was clearly an outlier in the diagram. Actually, ocean cruiser Queen Mary 2 arrived at New York Manhattan 

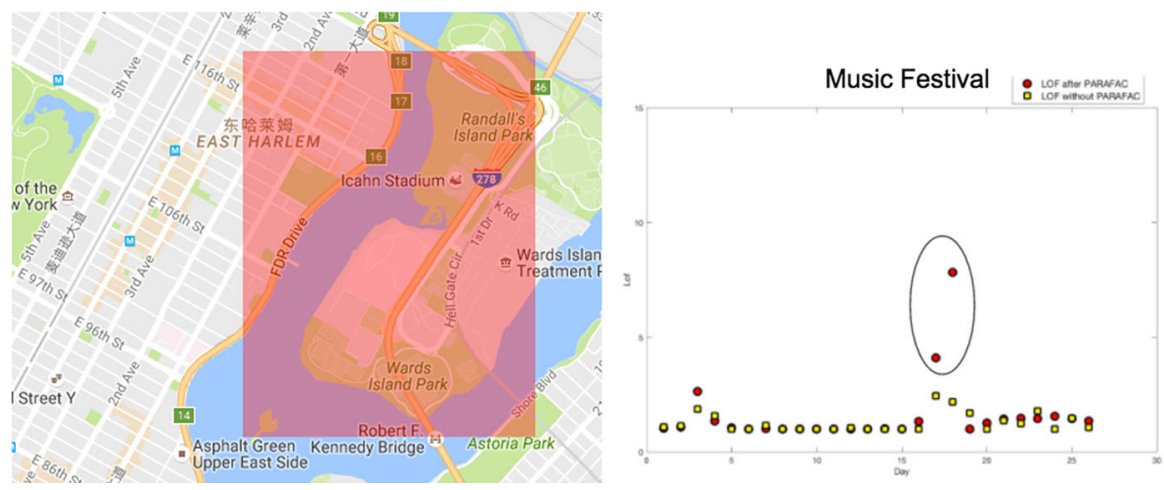

Fig. 3 Anomalous region and anomaly score diagram on August 30
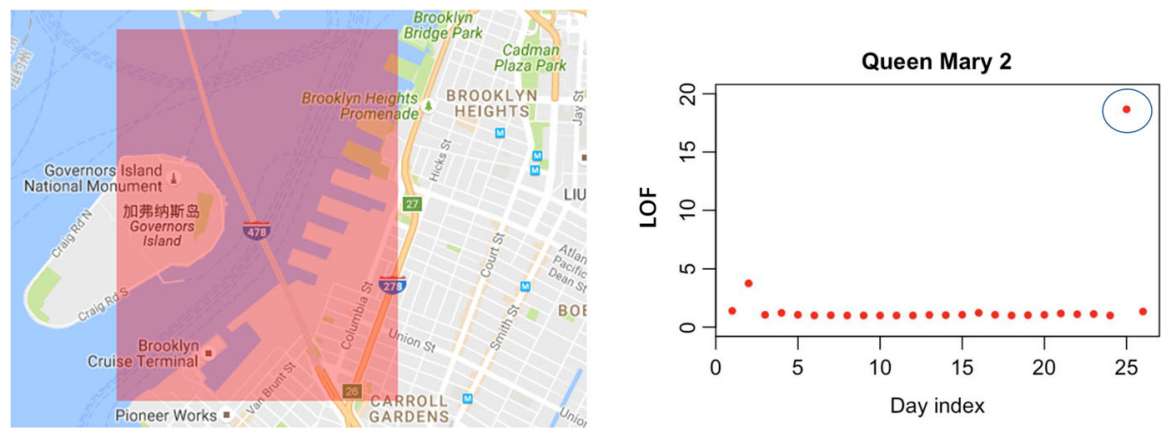

Fig. 4 Anomalous region and anomaly score diagram on September 27

Cruiser Terminal on that day, while she used to dock at New York Brooklyn Cruiser Terminal, which is located at the red area. Thus, we thought that the unusual docking terminal forced many people to travel from Brooklyn to Manhattan.

U.S. OPEN (Tennis Tournament) Semifinal and Final We observed a high anomaly score on September 6 and 7 in the region surrounding Flushing Meadows-Corona Park as shown in Fig. 5. For all Sundays and Saturdays in the third season of 2014, we can clearly find the anomaly which is around September 6 through the diagram with an anomaly score of 12.22 .

After checking U.S. OPEN's schedule, we found out that men's singles semifinals was on September 6 and women's singles final was on September 7. These matches involved famous tennis players such as Novak Djokovic, Roger Federer and Serena Williams. That would be reason why people were gathering there at a certain time. Except for the tournament, there existed another anomaly point with a score of 5.65 in the following week. This anomalous point indicated another special event in the park, which was the World Maker Faire on September 20 according to our search result. 

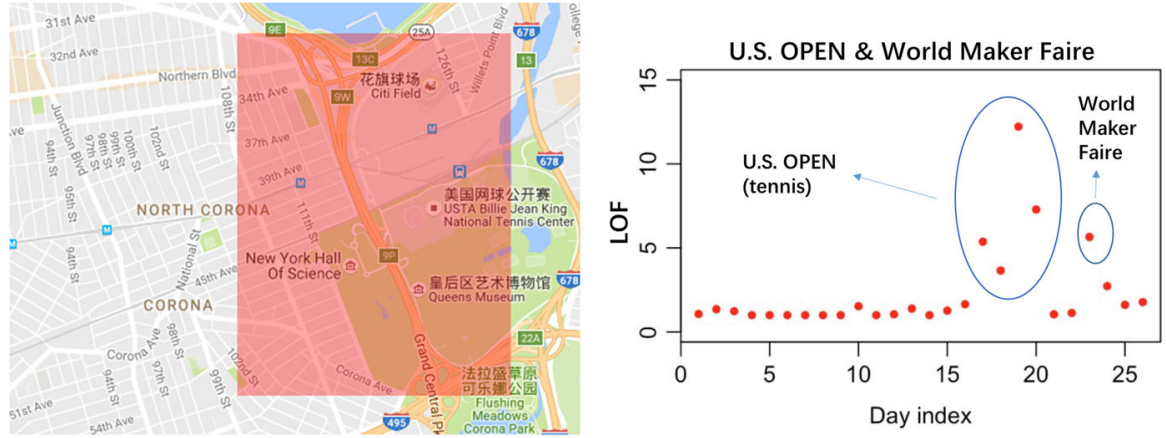

Fig. 5 Anomalous region and anomaly score diagram on September 6 and 7
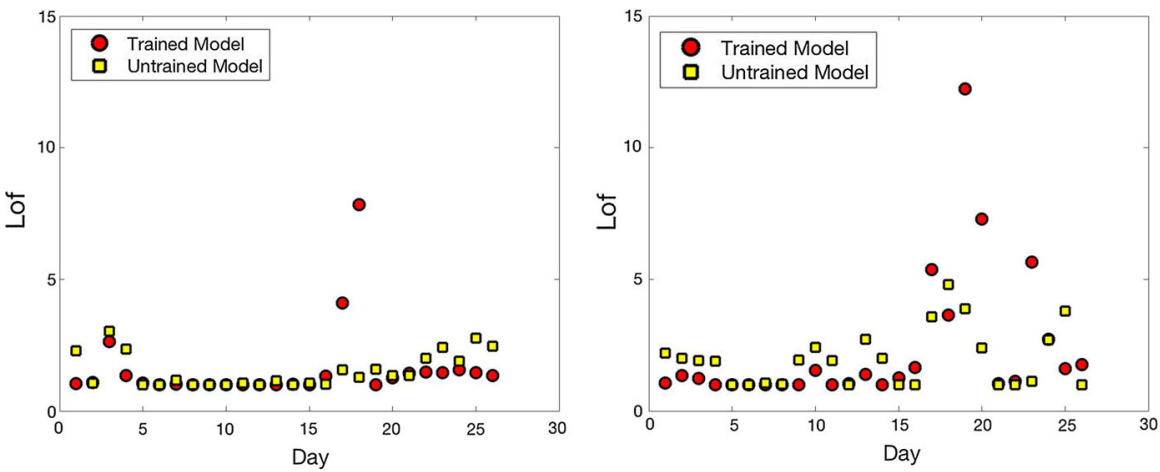

Fig. 6 Our model successfully detect the Music Festival (Left) and the U.S.OPEN (Right). And the untrained model cannot detect them and shows much more irregular results

\subsection{Compared with untrained model}

We have compared our TBAD framework with existing approach using untrained model. As untrained models share no similarity in factor matrices, the latent mobility patterns extracted from the tensor may differ a lot. Thus, the feature vectors extracted from the factor matrix $A$ have no baseline meaning and cannot be compared altogether. We have done experiments and the result in Fig. 6 shows that untrained model may produce massive wrong detection results.

\subsection{Compared with LOF}

We have also compared our method with the existing approach using LOF. Since LOF algorithm detects anomaly in numerous points and a point can only denote one time slice, time dimension is not considered, resulting in the insensitivity to the change in a dynamic traffic system.

We have done some comparison to demonstrate the idea. The result is shown in Fig. 3. The red and yellow points correspond to the result of our model and LOF 
respectively. The figure shows that our method is much more sensitive and effective than directly using LOF algorithm. Although the LOF results still have two days sharing the highest score, it is quite similar to other points and thus difficult for us to distinguish.

\section{Conclusion}

In this paper, we present a novel framework for detecting anomalous event. It integrates tensor decomposition and LOF algorithm to localize anomaly in a given time interval. We demonstrate the power of TBAD through its application in New York City yellow taxi data. We have done a case study based on anomalous event found by our system and several comparison with other present techniques. In the future, we would apply the framework to more areas such as pollution monitoring and severe weather warning. Furthermore, we would design a multi-functional visualization system to make the anomaly visually shown to the users and let the users to interact with the system to further improve its accuracy.

\section{References}

Bahadori MT, Yu QR, Liu Y (2014) Fast multivariate spatio-temporal analysis via low rank tensor learning. In: Advances in neural information processing systems, pp 3491-3499

Bai Y, Tezcan J, Cheng Q, Cheng J (2013) A multiway model for predicting earthquake ground motion. In: ACIS international conference on software engineering, artificial intelligence, networking and parallel/distributed computing (SNPD), pp 219-224

Breunig MM, Kriegel HP, Ng RT, Sander J (2000) Lof: identifying density-based local outliers. ACM Sigmod Rec 29:93-104

Chandola V, Banerjee A, Kumar V (2009) Anomaly detection: a survey. ACM Comput Surv (CSUR) 41(3): 15

Chen Y, Zhou XS, Huang TS (2001) One-class svm for learning in image retrieval. IEEE Image Process $1: 34-37$

Cichocki A, Zdunek R, Phan AH, Amari SI (2009) Nonnegative matrix and tensor factorizations: applications to exploratory multi-way data analysis and blind source separation. Wiley, New York

Fanaee-T H, Gama J (2015) Eigenevent: an algorithm for event detection from complex data streams in syndromic surveillance. Intell Data Anal 19(3):597-616

Fanaee-T H, Gama J (2016a) Tensor-based anomaly detection: an interdisciplinary survey. Knowl Based Syst 98:130-147

Fanaee-T H, Gama J (2016b) Event detection from traffic tensors: a hybrid model. Neurocomputing 203:2233

Fanaee-T H, Gama J (2014) An eigenvector-based hotspot detection. arXiv preprint arXiv:1406.3191

Gauvin L, Panisson A, Cattuto C (2014) Detecting the community structure and activity patterns of temporal networks: a non-negative tensor factorization approach. PloS ONE 9(1):e86028

Jiang M, Cui P, Faloutsos C (2016) Suspicious behavior detection: current trends and future directions. IEEE Intell Syst 31(1):31-39

Kim J, He Y, Park H (2014) Algorithms for nonnegative matrix and tensor factorizations: a unified view based on block coordinate descent framework. J Global Optim 58(2):285-319

Kolda TG, Bader BW (2009) Tensor decompositions and applications. SIAM Rev 51(3):455-500

Kotsia I, Guo W, Patras I (2012) Higher rank support tensor machines for visual recognition. Pattern Recogn 45(12):4192-4203

Liu S, Cui W, Wu Y, Liu M (2014) A survey on information visualization: recent advances and challenges. Visual Comput 30(12):1373-1393 
Liu D, Weng D, Li Y, Bao J, Zheng Y, Qu H, Wu Y (2017) SmartAdP: Visual analytics of large-scale taxi trajectories for selecting billboard locations. IEEE Trans. Vis. Comput. Graphics 23(1):1-10

Liu Y, Zhou B, Chen F, Cheung DW (2016) Graph topic scan statistic for spatial event detection. In: Proceedings of the 25th ACM International on Conference on Information and Knowledge Management. ACM, pp 489-498

Mao HH, Wu CJ, Papalexakis EE, Faloutsos C, Lee KC, Kao TC (2014) Malspot: Multi2 malicious network behavior patterns analysis. In: Pacific-Asia conference on knowledge discovery and data mining. Springer, pp 1-14

Matsubara Y, Sakurai Y, Faloutsos C, Iwata T, Yoshikawa M (2012) Fast mining and forecasting of complex time-stamped events. In: Proceedings of the ACM SIGKDD international conference on Knowledge discovery and data mining, pp 271-279

Nomikos P, MacGregor JF (1994) Monitoring batch processes using multiway principal component analysis. AIChE J 40(8):1361-1375

Papalexakis EE, Faloutsos C, Sidiropoulos ND (2012) Parcube: sparse parallelizable tensor decompositions. In: Joint European conference on machine learning and knowledge discovery in databases. Springer, pp 521-536

Papalexakis E, Pelechrinis K, Faloutsos C (2014) Spotting misbehaviors in location-based social networks using tensors. In: Proceedings of the international conference on world wide web. ACM, pp 551-552

Paschalidis IC, Smaragdakis G (2009) Spatio-temporal network anomaly detection by assessing deviations of empirical measures. IEEE/ACM Trans Netw (TON) 17(3):685-697

Prada MA, Dominguez M, Barrientos P, Garcia S (2012a) Dimensionality reduction for damage detection in engineering structures. Int J Mod Phys B 26(25):1246004

Prada MA, Toivola J, Kullaa J, HollméN J (2012b) Three-way analysis of structural health monitoring data. Neurocomputing 80:119-128

Rendle S (2012) Factorization machines with libfm. ACM Trans Intell Syst Technol (TIST) 3(3):57

Rozenshtein P, Anagnostopoulos A, Gionis A, Tatti N (2014) Event detection in activity networks. In: Proceedings of the 20th ACM SIGKDD international conference on Knowledge discovery and data mining. ACM, pp 1176-1185

Shi L, Gangopadhyay A, Janeja VP (2015) Stensr: spatio-temporal tensor streams for anomaly detection and pattern discovery. Knowl Inf Syst 43(2):333

Sun GD, Liang R, Qu H, Wu Y (2017a) Embedding spatiotemporal information into maps by route-zooming. IEEE Trans. Vis. Comput. Graphics 23(5):1506-1519

Sun G, Tang T, Peng TQ, Liang R, Wu Y (2017b) Socialwave: visual analysis of spatio-temporal diffusion of information on social media. ACM Trans Intell Syst Technol 9(2):15

Sun J, Tao D, Papadimitriou S, Yu PS, Faloutsos C (2008) Incremental tensor analysis: Theory and applications. ACM Trans Knowl Discov Data (TKDD) 2(3):11

Sun J, Tao D, Faloutsos C (2006) Beyond streams and graphs: dynamic tensor analysis. In: Proceedings of the 12th ACM SIGKDD international conference on Knowledge discovery and data mining. ACM, pp 374-383

Sun G, Wu YC, Liang RH, Liu SX (2013) A survey of visual analytics techniques and applications: stateof-the-art research and future challenges. J Comput Sci Tech 28(5):852-867

Tao D, Li X, Hu W, Maybank S, Wu X (2005) Supervised tensor learning. In: IEEE international conference on data mining

Thai-Nghe N, Horváth T, Schmidt-Thieme L (2010) Factorization models for forecasting student performance. In: Educational Data Mining 2011

Tian X, Zhang X, Deng X, Chen S (2009) Multiway kernel independent component analysis based on feature samples for batch process monitoring. Neurocomputing 72(7):1584-1596

Tork HF, Oliveira M, Gama J, Malinowski S, Morla R (2012) Event and anomaly detection using tucker3 decomposition. In: Workshop on ubiquitous data mining, p 8

Wang XR, Lizier JT, Obst O, Prokopenko M, Wang P (2008) Spatiotemporal anomaly detection in gas monitoring sensor networks. In: Wireless sensor networks: 5th European conference, EWSN 2008. Springer, pp 90-105

Wang J, Gao F, Cui P, Li C, Xiong Z (2014) Discovering urban spatio-temporal structure from time-evolving traffic networks. In: Asia-Pacific web conference. Springer, pp 93-104

Weng D, Zhu H, Bao J, Zheng Y, Wu Y (2018) Homefinder revisited: finding ideal homes with reachability centric multi-criteria decision making. In Proceedings of ACM CHI 
Wu Y, Lan J, Shu X, Ji C, Zhao K, Wang J, Zhang H (2018) ITTVIS: Interactive visualization of table tennis data. IEEE Trans Visualization and Comp Graphics 24(1):709-718

Xia J, Chen W, Hou Y, Hu W, Huang X, Ebertk DS (2016) DimScanner: A relation-based visual exploration approach towards data dimension inspection. In: IEEE conference on visual analytics science and technology (VAST). pp 81-90

Xie C, Chen W, Huang X, Hu Y, Barlowe S, Yang J (2014) VAET: A visual analytics approach for etransactions time-series. IEEE Trans. Vis. Comput. Graphics 20(12):1743-1752

Xu P, Mei H, Ren L, Chen W (2017) ViDX: Visual diagnostics of assembly line performance in smart factories. IEEE Trans. Vis. Comput. Graphics 23(1):291-300

Young WC, Blumenstock JE, Fox EB, McCormick TH (2014) Detecting and classifying anomalous behavior in spatiotemporal network data. In: Proceedings of KDD workshop on learning about emergencies from social information (KDD-LESI 2014), pp 29-33

Yuan J, Zheng Y, Xie X (2012) Discovering regions of different functions in a city using human mobility and pois. In: Proceedings of the ACM SIGKDD international conference on Knowledge discovery and data mining, pp 186-194

Zhang T, Wang X, Li Z, Guo F, Ma Y, Chen W (2017) A survey of network anomaly visualization. Sc China Infor Sci 60(12):121101

Zhao Z, Cheng Z, Hong L, Chi EH (2015) Improving user topic interest profiles by behavior factorization. In: Proceedings of the international conference on world wide web. ACM, pp 1406-1416

Zheng Y, Liu T, Wang Y, Zhu Y, Liu Y, Chang E (2014) Diagnosing New York city's noises with ubiquitous data. In: Proceedings of the ACM international joint conference on pervasive and ubiquitous computing, pp 715-725 